\title{
Pulse steroid therapy in rheumatoid arthritis: Can equivalent doses of oral prednisolone give similar clinical results to intravenous methylprednisolone?
}

\author{
MALCOLM D SMITH, MICHAEL J AHERN, \\ PETER J ROBERTS-THOMSON
}

From the Department of Immunology and Rheumatology, Flinders Medical Centre; and the Repatriation General Hospital, Adelaide, South Australia

SUMmARY Pulse methylprednisolone therapy has dramatic effects on clinical and immunologicåㅇ parameters of disease activity in patients with rheumatoid arthritis. Previous studies of this treatment have all used the intravenous route and methylprednisolone succinate. This study addresses the question of whether oral prednisolone in equivalent doses can substitute fo intravenous methylprednisolone in pulse therapy in a double blind parallel study. It is shown tha oral prednisolone has clinical and immunological effects equivalent to those of intravenous methylprednisolone, making it possible to administer pulse therapy to patients with rheumatoi $\$$ arthritis as outpatients without the inconvenience and inherent dangers of intravenous administration.

Pulse methylprednisolone therapy has been used for several years as a treatment for active synovitis for patients with rheumatoid arthritis (RA). ${ }^{1-6}$ Previous studies have examined the response to conventional doses of oral prednisolone or 'megadoses' of intravenous methylprednisolone, but no studies have considered whether equivalent doses of oral prednisolone would produce the same clinical effects as megadoses of methylprednisolone administered intravenously. We therefore examined the clinical response and side effects resulting from equivalent oral and intravenous doses of corticosteroids in a double blind manner to see whether oral pulse therapy was an effective substitute for intravenous pulse therapy in rheumatoid arthritis.

\section{Patients and methods}

Twenty four patients with definite rheumatoid arthritis (American Rheumatism Association criteria) and active joint disease were prospectively entered into the study and randomly assigned to two patient groups. Clinical details of both patient groups are presented in Table 1; four patients in

Accepted for publication 23 June 1987.

Correspondence to Dr Malcolm D Smith, Department of Immunology, Flinders Medical Centre, Bedford Park, South Australia 5042. group 1 and five patients in group 2 started with new suppressive agent at the time of receiving pulse

Table 1 Clinical details of patients randomly assigned to two groups

\begin{tabular}{|c|c|c|c|}
\hline & Group 1 & Group 2 & \\
\hline Number of patients & 12 & 12 & \\
\hline Mean age (range) & $58 \cdot 6(42-69)$ & $62 \cdot 4(48-74)$ & \\
\hline $\operatorname{Sex}(M: F)$ & $5: 7$ & $4: 8$ & \\
\hline $\begin{array}{l}\text { Mean disease duration } \\
\text { (years) }\end{array}$ & $6 \cdot 4(3-10)$ & $7 \cdot 2(2-12)$ & \\
\hline \multicolumn{4}{|l|}{ Drug treatment } \\
\hline NSAIDs* & 12 & 11 & \\
\hline Gold & 3 & 5 & \\
\hline D-Penicillamine & 2 & 1 & \\
\hline Sulphasalazine & 2 & 1 & \\
\hline VAS pain $(\mathrm{cm})^{*}$ & $7 \cdot 0(0.6) \dagger$ & $6.9(2)$ & \\
\hline VAS mobility $(\mathrm{cm})$ & $6 \cdot 1(0 \cdot 6)$ & $6.4(0.4)$ & \\
\hline \multicolumn{4}{|l|}{ Duration of morning } \\
\hline stiffness (min) & $228(32)$ & $238(30)$ & \\
\hline Articular index & $26(2 \cdot 2)$ & $27 \cdot 6(2 \cdot 6)$ & \\
\hline $\operatorname{ESR}(\mathrm{mm} / \mathrm{h})^{*}$ & $56 \cdot 7(10)$ & 74.9 (9) & \\
\hline CRP (mg/l)* & $64 \cdot 6(17 \cdot 8)$ & $94.9(17.5)$ & \\
\hline Rheumatoid factor (IU/ml) & $1297(611)$ & $2014(810)$ & \\
\hline \multicolumn{4}{|l|}{ Immune complexes } \\
\hline $\begin{array}{l}\text { (units/ml) } \\
\text { Pulse treatment given }\end{array}$ & $\begin{array}{l}27 \cdot 6(8 \cdot 6) \\
\text { Active oral }\end{array}$ & $\begin{array}{l}36 \cdot 2(9 \cdot 6) \\
\text { Active intra }\end{array}$ & \\
\hline
\end{tabular}

*NSAIDs=non-steroidal anti-inflammatory drugs; VAS=visu analogue score; $\mathrm{ESR}=$ erythrocyte sedimentation rate; $\mathrm{CRP}=\mathrm{E}$ reactive protein.

+Values are mean (SEM). 
therapy. There were no significant differences between the two treatment groups at the time of entry into the study. Clinical assessments were made before the start of the study and at 2, 4, 8, 12, 16, 20, and 24 weeks after receiving pulse therapy. Clinical parameters assessed were visual analogue scale for pain (VAS pain) and mobility (VAS mobility), duration of morning stiffness, grip strength $(\mathrm{mmHg})$, and articular index (Ritchie method). Laboratory parameters were assessed at the same time as clinical parameters and included $C$ reactive protein (CRP), erythrocyte sedimentation rate (ESR), rheumatoid factor, circulating immune complex levels (by $\mathrm{C} 1 \mathrm{q}$ liquid phase assay), ${ }^{7}$ and immunoglobulin levels (IgG, $\operatorname{IgM}$, and $\operatorname{IgA}$ ). Neutrophil and lymphocyte counts were measured as well as $\mathrm{T}$ cell markers (helper subset, supressor subset, class II major histocompatibility complex (MHC) positive cells) and $B$ cells using monoclonal antibodies and standard immunofluorescence techniques on mononuclear cells prepared on FicollHypaque gradients. ${ }^{8}$ Patients were included in the study if the following criteria were met: definite rheumatoid arthritis of at least six months' duration, articular index greater than 10, CRP estimation greater than twice the normal range (normal $<6$ $\mathrm{mg} / \mathrm{l})$, ESR greater than twice the upper limit of normal (normal $<10 \mathrm{~mm} / \mathrm{h}$ ), and duration of morning stiffness exceeding 60 minutes.

Patients were given both an intravenous infusion (over 40 minutes by an infusion pump) and oral medication, with only one of these containing the active treatment. The active medication was given as $1 \mathrm{~g}$ intravenous methylprednisolone succinate or $1 \mathrm{~g}$ oral prednisolone $(40 \times 25 \mathrm{mg}$ tablets $)$. All medications were prepared and administered in a double blind fashion with matching placebos consisting of either compound lactose tablets or $100 \mathrm{ml}$ intravenous saline. Patients were given the same treatment at the same time in the morning on three successive days.

Response was defined as a greater than $50 \%$ reduction in three or more of the clinical parameters of disease activity and relapse was defined as an increase of greater than $30 \%$ in three or more of the clinical parameters of disease activity compared with the lowest value for each parameter before or after treatment. The time of relapse was defined as that time when the first clinical parameter increased to greater than $30 \%$ above the lowest value obtained.

S T A T IS T I C S

Analysis of between group differences was performed by the Scheffe method of one way analysis of variance.

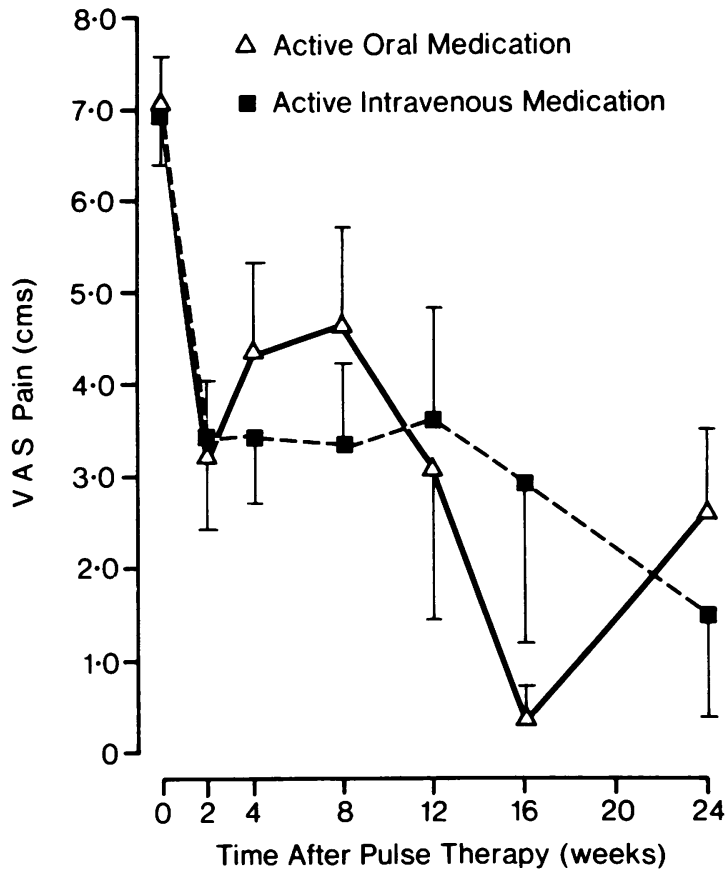

Fig. 1 Effect of oral and intravenous pulse therapy on visual analogue score for pain. Error bars indicate standard error of the mean.

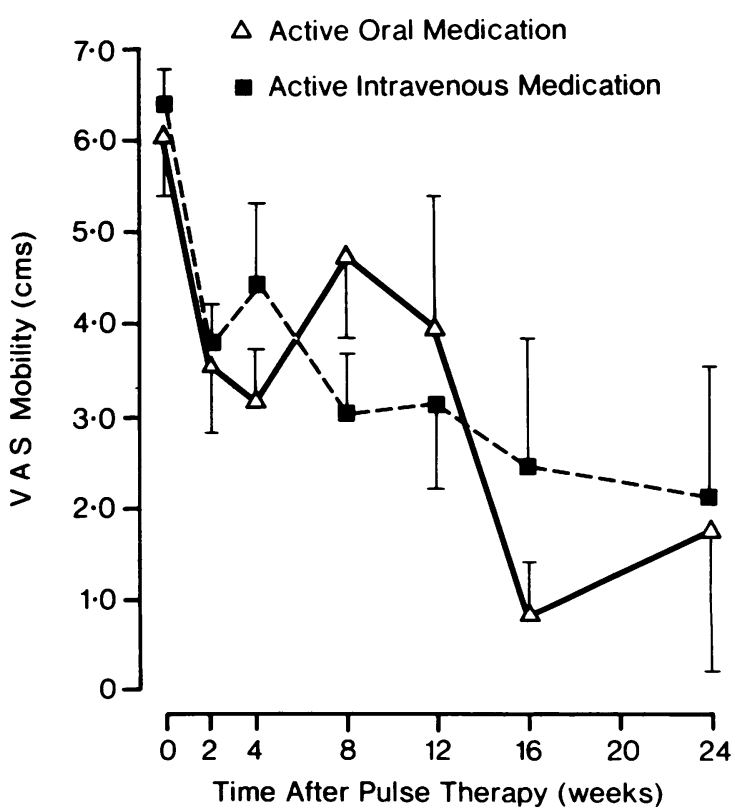

Fig. 2 Effect of oral and intravenous pulse therapy on visual analogue score for mobility. Error bars indicate standard error of the mean. 
Analysis of within group effects with time was performed using a multiple analysis of variance and Bonferroni's method for multiple comparisons.

Statistical significance was accepted at the $5 \%$ level.

An estimate of the $\beta$ error in the study was made. ${ }^{9}$ With a $90 \%$ response rate in the intravenous methylprednisolone group and a significance of $5 \%$, we would not be able to detect any less than a $35 \%$ difference between the two treatment groups in this study.

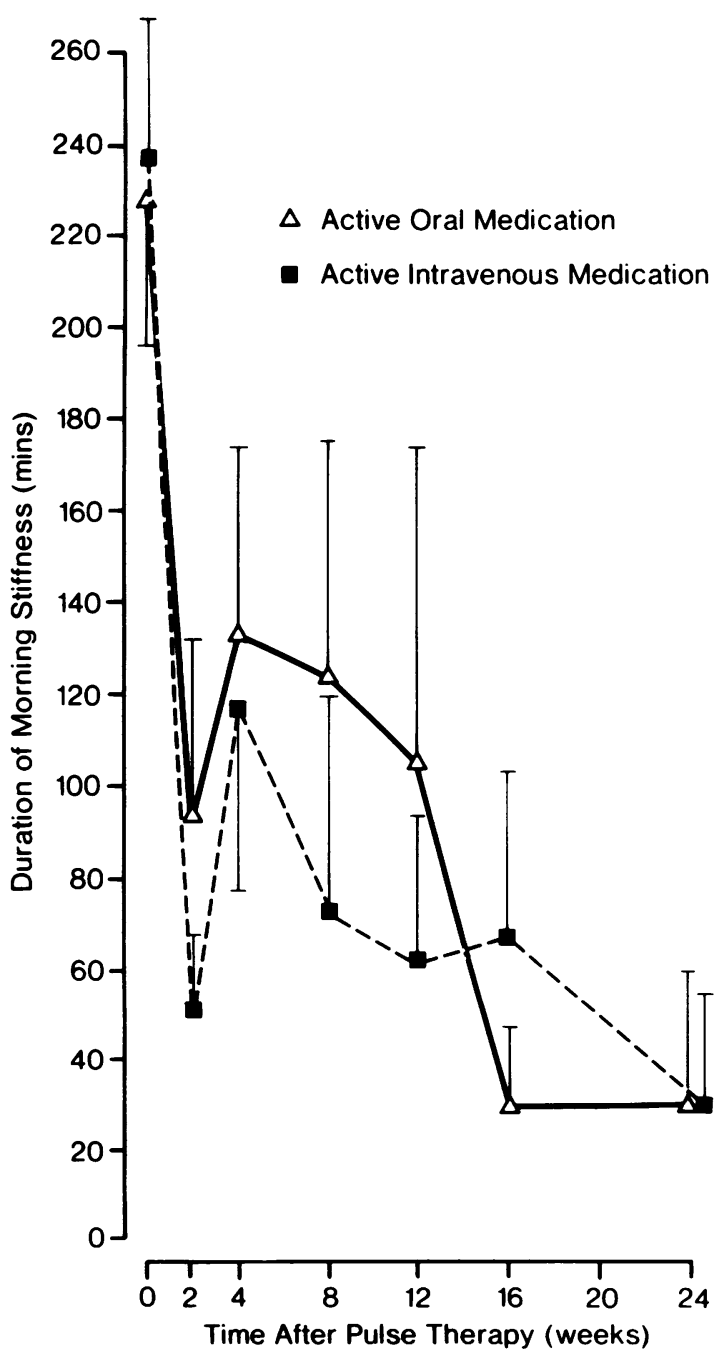

Fig. 3 Effect of oral and intravenous pulse therapy on duration of morning stiffness. Error bars indicate standard error of the mean.
Results

There were 12 patients in each treatment group, on group receiving intravenous methylprednisolone and placebo tablets, while the other group receive oral prednisolone and intravenous saline. Mea duration of response was 13 weeks in the grouf receiving the active oral preparation and 13.6 weeks in the group treated with the active intravenous preparation. Two patients in each group had not relapsed at completion of the study and one patierp in the active oral preparation group had a duration of response of less than four weeks.

The effects of pulse therapy on the clinical ant laboratory parameters of disease activity and on neutrophil and lymphocyte counts and cell surface markers are presented in Figs 1-7 and Tables 2 to $\$$

Analysis of effect of time, within groups, showed significant changes $(p<0.05)$ in VAS pain and mobility, duration of morning stiffness, gri strength, and articular index up to 16 weeks follo up compared with pretreatment levels in both groups. CRP, immune complexes, IgA, and rheumatoid factor levels were also significantfy decreased in both groups up to eight weeks follow up in both groups, but no significant change was seen in either group for IgG and IgM levels or fo neutrophil and lymphocyte counts when compares with pretreatment levels. There were no significan

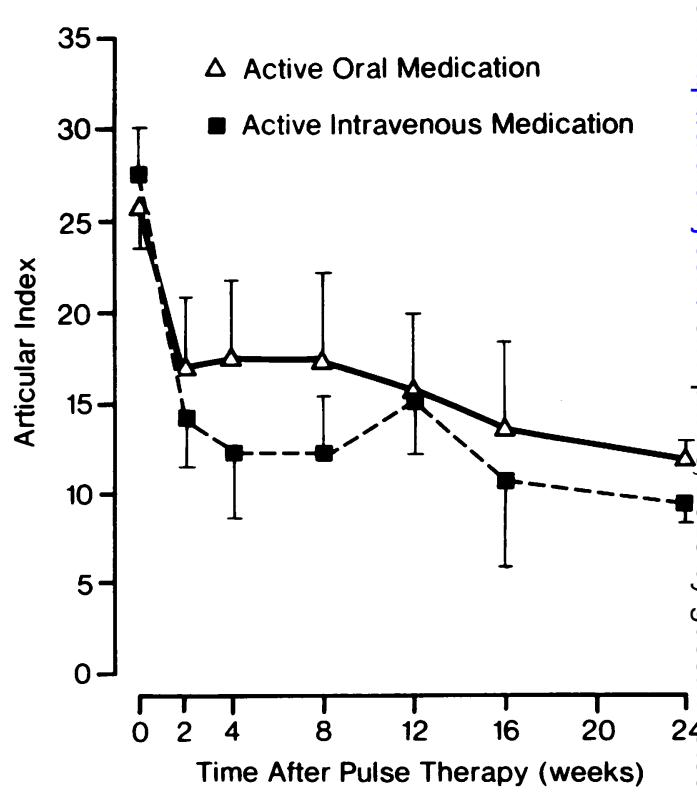

Fig. 4 Effect of oral and intravenous pulse therapy on articular index. Error bars indicate standard error of the mean. 
changes in any of the lymphocyte subsets measured in either group at any period of follow up when compared with pretreatment values.

Between group assessments did not show any significant difference between the two treatments at any of the follow up times for any of the clinical or laboratory parameters measured, though there was a trend towards greater response in the group treated with intravenous methylprednisolone.

Side effects were minimal with either treatment, though one patient had a gastrointestinal haemorrhage four weeks after receiving intravenous methylprednisolone succinate which may have been related to the pulse therapy or the non-steroidal anti-inflammatory drug (NSAID), sulindac, concurrently being taken. One patient developed an infection as a direct result of the intravenous method of treatment.

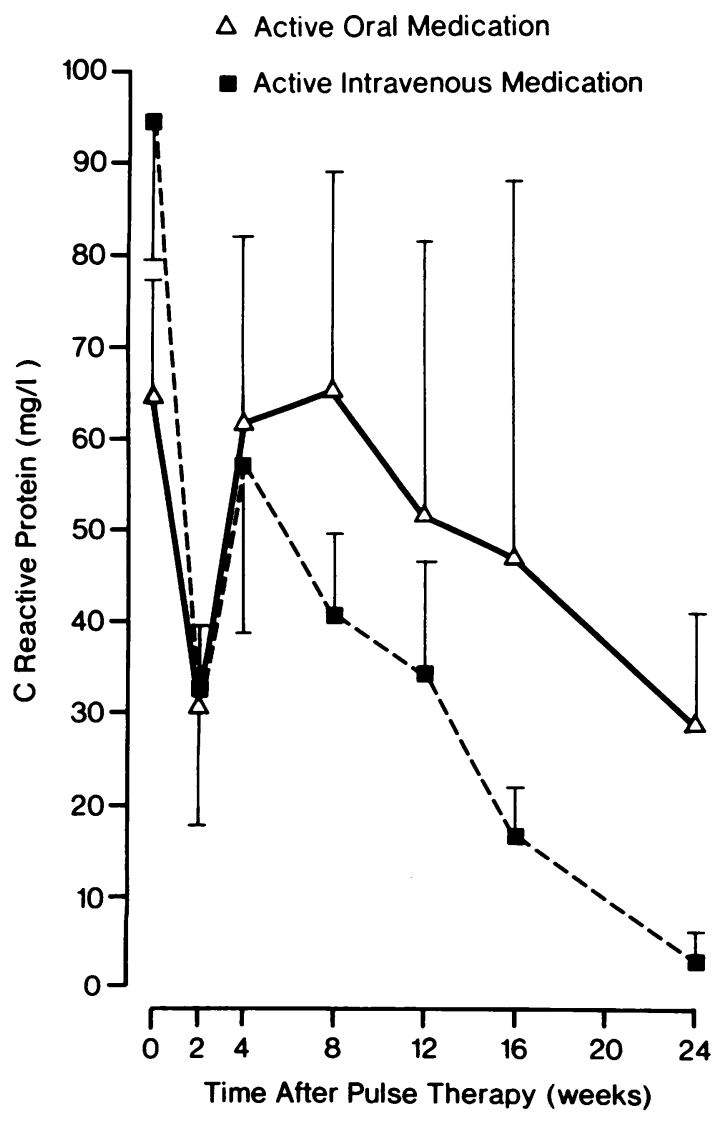

Fig. 5 Effect of oral and intravenous pulse therapy on $C$ reactive protein. Error bars indicate standard error of the mean.

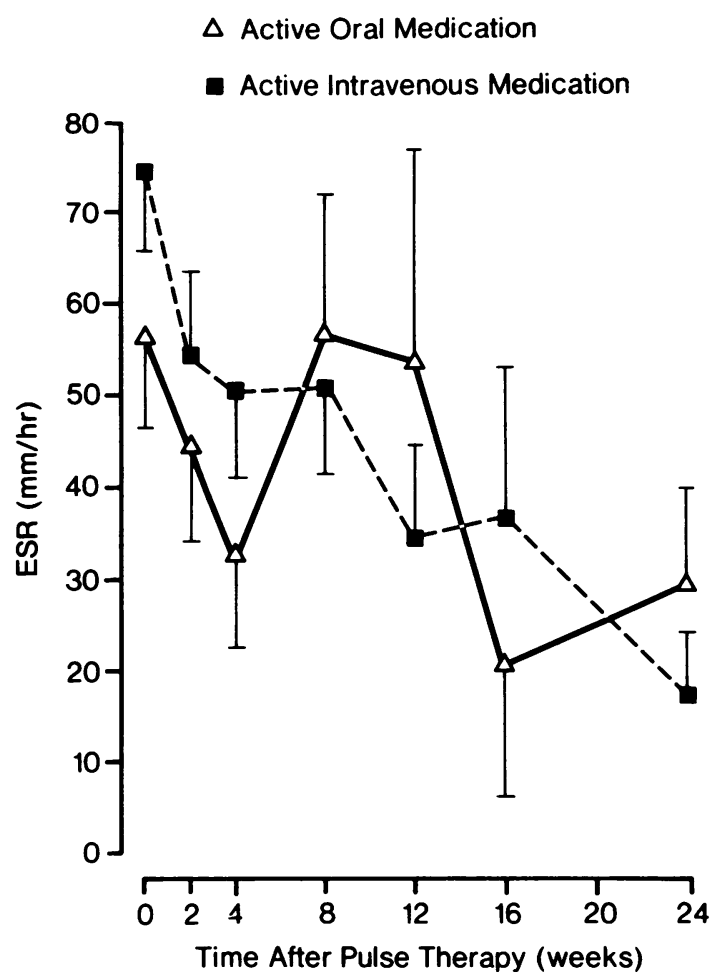

Fig. 6 Effect of oral and intravenous pulse therapy on erythrocyte sedimentation rate. Error bars indicate standard error of the mean.

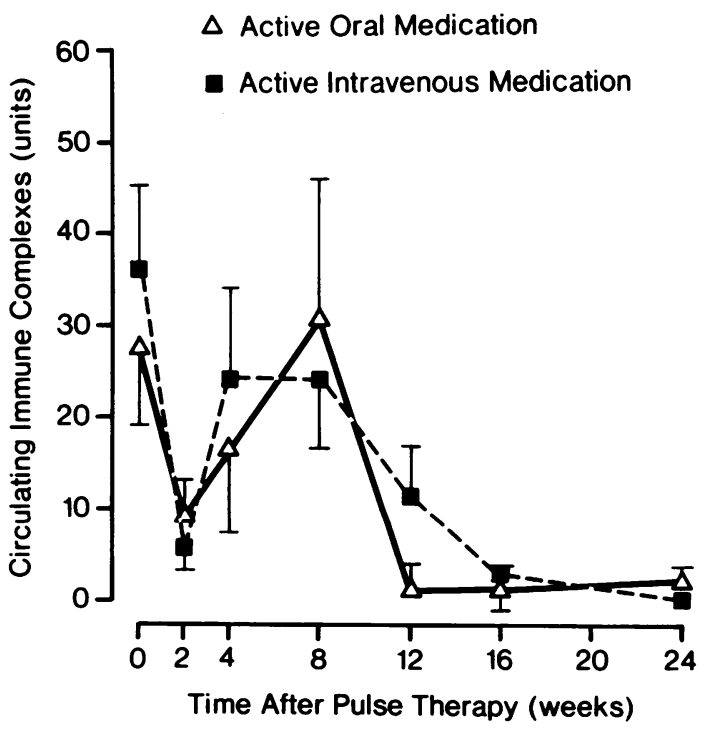

Fig. 7 Effect of oral and intravenous pulse therapy on circulating immune complex levels. Error bars indicate standard error of the mean. 
32 Smith, Ahern, Roberts-Thomson

Table 2 Effect of pulse therapy on rheumatoid factor and immunoglobulin levels

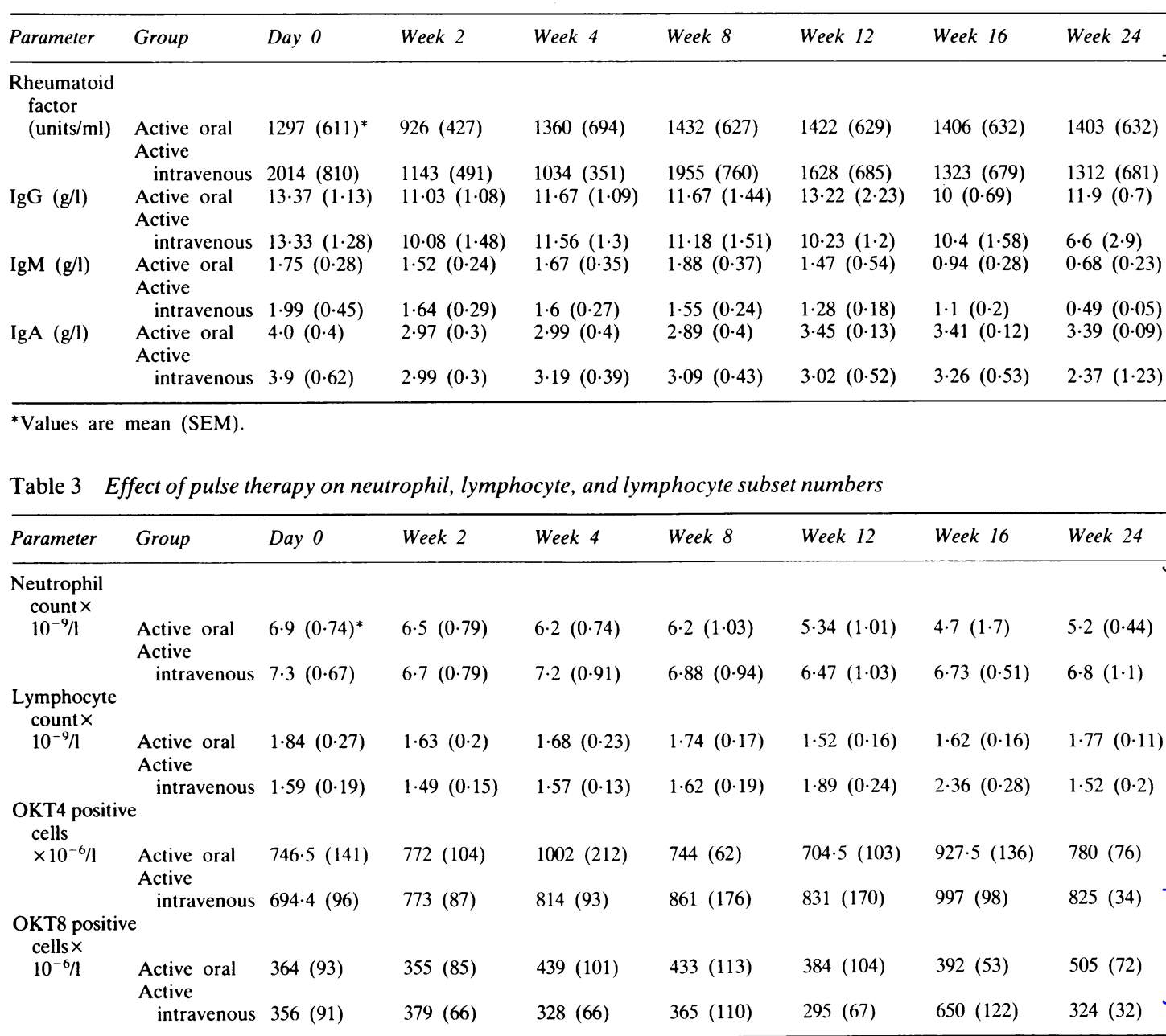

*Values are mean (SEM).

Table 4 Effect of pulse therapy on lymphocyte subsets

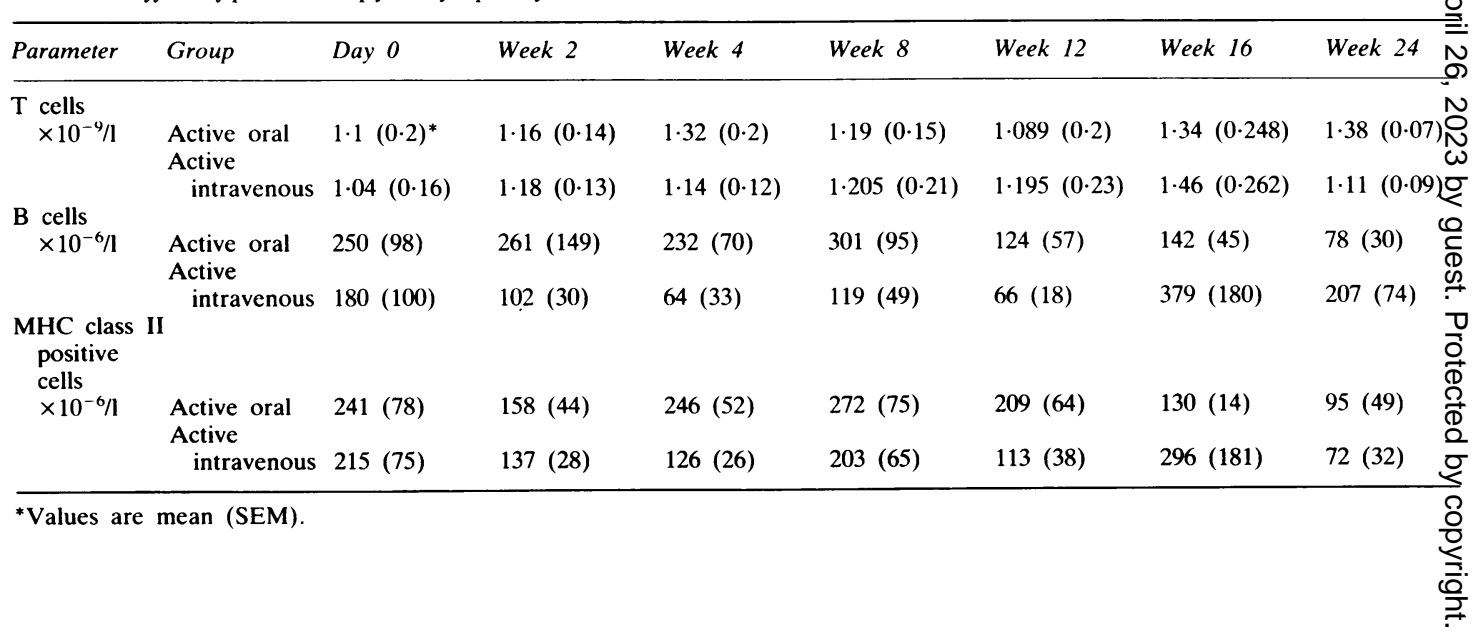




\section{Discussion}

Several authors have shown that intravenous pulse methylprednisolone therapy has a significant but temporary beneficial effect on the synovitis of patients with active RA. ${ }^{1-6}$ We have also shown this significant improvement in patients treated not only with pulse therapy with intravenous methylprednisolone but also with oral prednisolone. This study suggests that equivalent doses of oral prednisolone and intravenous methylprednisolone have equivalent clinical and immunological effects, but has not established the minimum dose of either treatment required to achieve this response. Although it is possible that larger patient numbers in each treatment group would have demonstrated a statistically significant difference in favour of the intravenous pulse, it appears unlikely from this study that this would outweigh the obvious clinical advantages of oral pulse therapy. Side effects were minimal with either treatment despite the theoretical concerns of high doses of oral corticosteroids on the upper gastrointestinal tract mucosa. ${ }^{10}$ Some doubt has recently been cast on the association of corticosteroid treatment with gastrointestinal tract ulceration, ${ }^{11-13}$ and there was no evidence of this side effect in patients who received oral prednisolone pulse therapy. Most studies examining the relation between steroid therapy and peptic ulcer have involved much lower doses of oral corticosteroids than those used in this study and there is little information in the literature on peptic ulcer complications when large doses of oral prednisolone or intravenous methylprednisolone are administered. As no significant difference was detected between the two treatment groups with respect to duration of remission or effects on the clinical and laboratory parameters of disease activity measured in this study it appears that oral prednisolone can safely and effectively replace intravenous methylprednisolone in pulse therapy for RA. This would remove the additional hazards of intravenous cannulation and administration and allow pulse therapy to be conducted as an outpatient procedure with less interference to patients and fewer demands on medical and nursing resources.

We thank Dr R Geddes and Dr W Hill for contributing patients to this study, Mrs J Smith for statistical advice, and Miss B Roylance for typing the manuscript. M D Smith is a grateful recipient of an NH and MRC postgraduate scholarship. This study was supported in part by a grant from the Department of Veterans Affairs.

\section{References}

1 Liebling $M$. Pulse methylprednisolone therapy in active rheumatoid arthritis. Internal Medicine for the Specialist 1983; 4: No 8.

2 Barry $M$. The use of high dose pulse methylprednisolone in rheumatoid arthritis. Arch Intern Med 1985; 145: 1483-4.

3 Liebling M R, Leib E, McLaughlin K, et al. Pulse methylprednisolone in rheumatoid arthritis; a double blind crossover trial. Ann Intern Med 1981; 94: 21-6.

4 Williams J A, Baylis E M, Shipley M E. A double blind placebo controlled trial of methylprednisolone pulse therapy in active rheumatoid disease. Lancet 1982; ii: 237-40.

5 Forster P J G, Grindulis K A, Neumann V, Hubball S, McConkey B. High dose intravenous methylprednisolone in rheumatoid arthritis. Ann Rheum Dis 1982; 41: 444-6.

6 Fan P T, Yu D T Y, Clements P J, Fowlston S, Eisman J, Bluestone R. Effect of corticosteroids on the human immune response: comparison of one and three daily $1 \mathrm{gm}$ intravenous pulses of methylprednisolone. J Lab Clin Med 1978; 91: 625-34.

7 Zubler R H, Nydegger U, Perrin L H, et al. Circulating and intra articular immune complexes in patients with rheumatoid arthritis. Correlation of ${ }^{125} \mathrm{I} \mathrm{Clq}$ binding activity with clinical and biological features of the disease. J Clin Invest 1976; 47: 1308-19.

8 Bertouch J V, Roberts-Thomson P J, Smith M D, et al. Methylprednisolone infusion therapy in rheumatoid arthritis patients. Arthritis Rheum 1986; 29: 32-8.

9 Macuch R, Sim R. Sample size requirements for evaluating a conservative therapy. Cancer Treat Rep 1978; 62: 1037-40.

10 Messer J, Reitman D, Sacks H S, Smith H Jr, Chalmers T C. Association of adrenocorticosteroid therapy and peptic ulcer disease. $N$ Engl J Med 1983; 309: 21-4.

11 Conn H O, Blitzer B L. Nonassociation of adrencorticosteroid therapy and peptic ulcer. $N$ Engl $J$ Med 1976; 294: 473-9.

12 Spiro H M. Is the steroid ulcer a myth? N Engl J Med 1983; 309: 45-7.

13 Conn H O, Poynard T. Adreno corticosteroid administration and peptic ulcer: a critical analysis. J Chronic Dis 1985; 38: 457-8. 